\title{
Review
}

Ophthalmic

Research

Ophthalmic Res 2013;50:197-208

DOI: $\underline{10.1159 / 000348736}$
Received: January 1, 2013

Accepted: January 14, 2013

Published online: September 11, 2013

\section{Glaucoma: Present Challenges and Future Trends}

\author{
Paolo Bettin Federico Di Matteo \\ Department of Ophthalmology, San Raffaele Scientific Institute, Milan, Italy
}

\section{Key Words}

Glaucoma · Detection - Genetics · Medical treatment ·

Surgery

\begin{abstract}
The authors indicate four key issues which, in their opinion, represent the most stimulating challenges in the field of glaucoma today, and describe the present approaches and the developments that can be expected or advised in the near future: (1) Glaucoma is often undiagnosed or diagnosed too late. Accurate detection is crucial to correctly recognize and treat affected subjects, thus reducing the disability and the social burden of the disease. Is glaucoma screening advisable and cost-effective? What strategies can be implemented to achieve an earlier diagnosis? (2) What role can genetics play in glaucoma clinics? (3) What are the fundamentals and the limits of medical hypotensive and nonhypotensive treatment of glaucoma today, and what novelties could be introduced in the next few years? (4) What are the current and future options for glaucoma surgery? Should we aim at reducing aqueous production or at enhancing aqueous outflow? What are the success figures and the pros and cons of $a b$ externo procedures with external filtration (trabeculectomy, nonpenetrating techniques and glaucoma drainage devices) and of ab externo techniques with internal filtration (viscocanalostomy and canaloplasty)? Will microin-
\end{abstract}

vasive procedures ever play a role in the treatment of the disease? These questions and the possible future developments in the management of glaucoma are discussed critically, based on the current clinical state of the art, on a review of the literature, and on an everyday experience in a busy university hospital glaucoma service.

Copyright $\odot 2013$ S. Karger AG, Basel

\section{Introduction}

What are the most stimulating challenges in the field of glaucoma today? How are they addressed now and how will they be addressed in the near future to ensure our patients a longer symptom-free life span and a better quality of life?

Glaucoma is a diffuse disease: it is the first cause of unemendable visual disability and blindness worldwide [1] and a recent epidemiological review concludes that 1 in 40 adults over 40 years of age suffers from glaucoma with visual loss [2]. Estimating a prevalence of $2.65 \%$ in the population over 40 , the overall number of glaucomatous subjects is expected to increase in the course of the present decade, owing both to demographic expansion and population ageing, from about 60 million in 2010 to nearly 80 million in 2020 [3]. One of the main factors that lead to visual impairment in glaucoma is late diagnosis and un-

\section{KARGER}

E-Mail karger@karger.com

www.karger.com/ore
(C) 2013 S. Karger AG, Basel

0030-3747/13/0504-0197\$38.00/0
Paulo Bettin

Department of Ophthalmology, University Vita-Salute, San Raffaele Scientific Institute Via Olgettina 60

IT-20132 Milan (Italy)

E-Mail bettin.paolo@hsr.it 
derdetection, with over half of the cases undiagnosed in the general population not only in emerging countries, but also in regions with high-level medical standards [4]: if more effective detection strategies are implemented, more cases will emerge and require treatment in the next years. Based on an everyday experience in glaucoma management, we individualize and discuss four key issues to improve glaucoma care with individual and social benefits:

(1) Appropriate detection of glaucoma

(2) Genetics and glaucoma

(3) Medical treatment of glaucoma

(4) Glaucoma surgery

\section{Appropriate Detection of Glaucoma}

Being generally asymptomatic for years, glaucoma is often diagnosed too late: half of the cases suffer from moderate to advanced disease in the worse eye upon first presentation even in nations with high sociosanitary standards $[5,6]$ and this heavily increases the human and economic burden of the disease for individuals and society [4]. Intraocular pressure (IOP) measurement is common in routine eye visits but is unfit to discriminate normal from glaucomatous eyes: up to half of the subjects with glaucoma may not have an elevated IOP upon examination and many ocular hypertensives do not need treatment and will never develop glaucoma in their lives. Glaucoma is undiagnosed in 50\% of cases in the western world, with higher figures in specific ethnicities, as demonstrated by several epidemiological studies (Baltimore Eye Survey, Rotterdam Eye Study, Blue Mountains Eye Study, Visual Impairment Project, Proyecto VER, and Latino Eye Study) and in up to $90 \%$ of cases in developing countries (Aravind Eye Study). On the other hand, glaucoma is paradoxically often overtreated: many subjects are treated although they have no real disease [7]. This strongly advocates a worldwide improvement in the accurate detection of the disease [8].

\section{Screening Strategies}

Tonometry performs very poorly, particularly in sensitivity, for glaucoma screening. Many other methods have been proposed, based on the study of eye morphology (optic nerve head, $\mathrm{ONH}$, ganglion cell or retinal nerve fiber layer, RNFL) and/or function. Some tests have been recognized as having higher performance, particularly some based on frequency-doubling perimetry, confocal scanning laser ophthalmoscopy and oculokinetic perimetry, but their recommendation for screening of the gen- eral population is supported by poor-quality evidence [9]. Unfortunately, the range of normality is broad (diseasefree subjects vary widely in functional and morphological testing), and nonglaucomatous conditions may be confounding, making the predictive power of screening tools proposed to date too low. Ideally, a good screening test is cheap, easy to administer, safe, quick, well accepted by probands and effectively discriminates normal from ill subjects. False negatives unacceptably delay proper diagnosis, thus increasing the burden of disease, and false positives generate direct costs due to unneeded further and more sophisticated testing. Presently, no single test can discriminate glaucoma from normality accurately enough to be effective for screening [10]. Moreover, most screening studies have been conducted on biased population samples with a high probability of excessive optimism in the evaluation of results [11]. There is therefore a substantial lack of evidence about the opportunity and costeffectiveness of mass glaucoma screening [12] and we even know too little about how to best minimize future visual disability [13]. In spite of this, targeted screening programs (in terms of age groups or selected populations) may be more efficient $[7,10]$ and could more effectively address multiple eye diseases simultaneously.

Our suggestions to improve the detection of glaucoma at relatively cheap social costs are the following:

(1) Positive family history is a strong risk indicator: blood relatives of glaucomatous patients should be advised to undergo specific glaucoma screening under the support of the health care systems.

(2) Cataract surgery is more and more diffuse and precocious in the developed world: most individuals undergo cataract extraction at least in one eye during their life and this is becoming a surrogate mass screening for eye comorbidities, particularly macular diseases and glaucoma. A preoperative visit with accurate diagnosis also has an ethical and medicolegal value in predicting the outcome of surgery and preventing future litigations.

(3) Nearly all adults in the developed world possess a driving license. In many countries the psychophysical fitness to drive must be certified by a medical examination and specific minimum visual capabilities are required, but the vision tests employed are unfit to detect glaucoma (in Italy most glaucomatous patients are regularly licensed because either they are unaware of the diagnosis or they omit to declare their condition). Driving licenses may expire periodically and can be banned due to driving accidents, motoring offences, substance abuse and physical or mental disease, and license renewal or end of ban often require a medical examination. In Italy, 
driving licenses expire at shorter intervals with age and a medical officer must certify compliance with minimum visual requirements as concerns spectacle-corrected visual acuity, stereopsis and color vision. This represents a unique screening opportunity, since the motivation to undergo and pass the test is strong. Moreover, population ageing is a relevant issue for road safety: periodical reevaluation of elderly subjects should be considered by state authorities, as well as for the sake of rule harmonization. If such medical examination is required, a complete eye visit including IOP measurement, gonioscopy, fundus examination and possibly a short functional test (e.g. frequency-doubling perimetry) could be enforced with minor extra costs. Many undiagnosed or suspect glaucomas and ocular hypertensives would be detected with individual and social benefits, not only because of timely disease management but also because glaucomatous drivers display a dramatically higher risk of causing road accidents (OR 7.2-12.4) [14] and missing peripheral hazards (OR 6.0) [15] compared to nonglaucomatous drivers, and this evidence should also raise the interest of insurance companies.

\section{Early Diagnosis}

Since general population screening for open-angle glaucoma (OAG) is not recommended [16], the diagnosis of asymptomatic glaucoma is mostly opportunistic. Subjects with early disease who do not undergo eye examination are obviously undiagnosed, but what is unacceptable is that the diagnosis may be missed in up to half of the cases even among subjects who do see ophthalmologists or optometrists for routine examinations [17].

Ophthalmoscopy with accurate $\mathrm{ONH}$ examination (with at least $\mathrm{ONH}$ drawing) should be mandatory in all routine visits, even in patients seen for simple spectacle prescription or generic checkup, so as to detect patients at risk and address them to further investigations. In case of manifest glaucoma with functional defects, conventional static perimetry is adequate for staging and followup, but initial or suspect glaucomas need further diagnostic steps since qualitative grading of the $\mathrm{ONH}$ damage has a too high intra- and interobserver variability even among glaucoma specialists [18]. A relationship between structure and function is necessarily present in glaucoma; the two aspects change together and mathematical modeling demonstrates that both tests may reveal a change, or either one may precede the other in detecting significant modifications [19]. However, in so-called preperimetric glaucomas, techniques studying the $\mathrm{ONH}$ and the retinal layers may be helpful to anticipate the appearance of

Glaucoma: Present Challenges and Future

Trends functional defects [20]. Several imaging devices have been recently developed to provide objective, quantitative, operator-independent measurements of the posterior pole, to compare a single test with a normative database and, even more usefully, to allow longitudinal analysis of series of tests acquired over time.

\section{Confocal Scanning Laser Ophthalmoscopy}

Each point of the ONH region is scanned by a focused laser beam. Incident light is reflected backwards through a confocal pinhole that suppresses light reflected or scattered from outside the focal plane. The commercially available system (Heidelberg Retina Tomograph) employs a $670-\mathrm{nm}$ diode laser to generate a stack of 64 parallel planes, each of which is composed of $384 \times 384$ pixels, covering a $15^{\circ}$ area of the peripapillary retina. Images are automatically aligned producing a 3-D tomographic map which is the basis for numeric computing of a series of morphometric parameters of the $\mathrm{ONH}$, some of which are generated based on previous manual identification of the $\mathrm{ONH}$ contour by the operator.

\section{Scanning Laser Polarimetry}

This system uses the birefringent properties of the ganglion cell axon microtubules to measure RNFL thickness. Within the commercially available scanning laser polarimetry system (GDx) a 780-nm laser beam is sent to the retinal plane and the change in polarization of the reflected beam is measured by a detector. A $256 \times 256$-pixel retardation image of the peripapillary retina is reconstructed and a 3.2-mm-wide circular RNFL scan centered on the $\mathrm{ONH}$ is automatically computed. As the birefringency of the cornea may be confounding, the most recent GDx versions include corneal compensation algorithms. Global and sectorial RNFL thickness measurements are provided along with a summary value (Nerve Fiber Index).

\section{Optical Coherence Tomography}

This technique produces cross-sectional images of the retina based on interferometry. A light source emits a beam which is split by a half-silvered mirror into two perpendicular beams: one travels through the eye and is reflected by ocular tissues and the other is reflected back by a mirror. The two beams are recombined and the pattern of constructive and destructive interference is analyzed to generate a tomographic image of the fundus. Time-domain systems use a moving reference mirror to analyze the depth ( $\mathrm{z}$ ) axis, and new-generation spectral-domain devices use Fourier transform to simultaneously compute 
the interference of a broad spectrum of incident and reflected light with a static reference arm, allowing better axial resolution and much faster acquisition time. More accurate segmentation algorithms allow direct measurement of individual retinal layers. Corrective optics techniques, such as adaptive optics, and higher resolution may allow visualization of single photoreceptor cells and details of the lamina cribrosa region [21].

\section{Novel Imaging Techniques}

New imaging methods for structural and dynamic assessment of $\mathrm{ONH}$ and retina have been recently proposed. Measurement of ocular blood flow using Doppler imaging has been used in experimental studies, although the poor reproducibility of the method limits its clinical applications. Doppler optical coherence tomography systems are under development and may soon provide a reliable and simple tool to evaluate blood flow in the $\mathrm{ONH}$.

Magnetic resonance imaging can be used to study optic pathways, geniculate bodies and visual cortex. Both structural and functional sequences can be used. Magnetic resonance imaging could become a powerful tool for research in glaucoma, but its clinical use is limited by high costs, long acquisition times, low resolution and reproducibility.

Despite technological progress, imaging methods will never be a substitute for ophthalmoscopy, but they can usefully complement it. Structural and functional tests must be chosen for each subject based on the specific case, and the results must be integrated in the clinical picture by a competent ophthalmologist gathering patient history and carrying out a complete eye examination. Without this basic approach, any instrumental test is just a waste of time and money for the patient and for the health care system. Moreover, some structural parameters such as $\mathrm{ONH}$ pallor and hemorrhage cannot be quantified by imaging methods, and nonglaucomatous conditions may affect visual function and must be recognized by an expert examiner. Biomicroscopy of the posterior pole and $\mathrm{ONH}$ is therefore still the mainstay in the glaucoma clinic, but quantitative analyses should be implemented if required.

The issue of glaucoma screening is controversial. The multiplication of costs involved in mass testing makes it non-cost-effective, but selected populations may benefit from targeted screening programs and common formalities, such as the ascertainment of fitness to drive, can offer an opportunity of cheap and diffuse testing.

\section{Genetics and Glaucoma}

Transmission of the disease occurs mostly in monogenic form in juvenile-onset OAG and in complex form in adults. It has been reported that $72 \%$ of primary OAG (POAG) cases have an inherited component [22].

More than 30 chromosomal loci have so far been implicated in POAG [23], but many of them have failed to be replicated across populations. In particular, 4 genes have been characterized: myocilin (MYOC) on GLC1A (1q32) [24], optineurin (OPTN) [25] on GLC1E (10p25), WDR36 on GLC1G (5q22.3) [26] and NTF4 on GLC1O (19q13.3) [27, 28]. In most cases, however, in spite of clear familial clustering, POAG does not follow a mendelian pattern of inheritance and mutations in these genes account for less than $10 \%$ of POAG overall [29].

Recently, in a genome-wide association study, a singlenucleotide polymorphism (SNP), rs4236601, near the caveolin1 (CAV1) and caveolin2 (CAV2) genes was identified to be strongly associated with POAG in the Icelandic population, and the association was replicated in multiple study populations [30]. In another recent genome-wide association study, SNPs at the transmembrane and coiledcoil domains 1 (TMCO1) and CDKN2B antisense RNA1 (CDKN2B-AS1) genes were found to be associated with POAG in different Caucasian cohorts [31].

Pseudoexfoliation glaucoma (PXG) is the most common identifiable form of secondary OAG worldwide. It is characterized by flakes of granular material at the pupillary margin of the iris and throughout the inner surface of the anterior chamber. PXG has been strongly associated with SNPs of the lysyl oxidase-like 1 (LOXL1) gene on chromosome 15q24.1 [32]. Two of these SNPs confer a higher than $99 \%$ population-attributable risk for pseudoexfoliation syndrome and PXG in the Nordic population; however, they carry different risks in different populations. The high-risk haplotypes also vary among distinct ethnicities [33-37]. LOXL1 is one of a group of enzymes involved in the cross-linking of collagen and elastin in the extracellular matrix. Its function in connective tissue maintenance has been confirmed in mice, but its actual role in PXG remains unclear.

The genetic factors determining IOP levels are largely unknown. Recent genome-wide association studies involving more than 19,000 subjects from the Netherlands, UK, Australia and Canada showed that IOP is significantly associated with two SNPs, rs11656696, located in GAS7 at 17p13.1, and rs7555523, located in TMCO1 at 1q24.1. In a meta-analysis of 4 case-control studies, both variants also showed evidence for association with glaucoma [38]. 
GAS7 and TMCO1 are highly expressed in the ciliary body and trabecular meshwork as well as in the lamina cribrosa, optic nerve and retina. Both genes functionally interact with known glaucoma disease genes. In the near future, if the sensitivity and specificity of genotyping increases and its cost decreases, it may become possible to screen individuals routinely for disease susceptibility.

\section{Medical Treatment of Glaucoma}

\section{Conventional (Hypotensive) Therapies}

Presently, glaucoma is treated with 5 classes of topical drugs that can also be used in association thanks to their complementary contribution to IOP reduction. Owing to their efficacy and tolerability, first-line drugs are $\beta$-blockers (BBs) and prostaglandin analogs (PGAs). BBs reduce IOP by decreasing aqueous formation; they do not affect pupil size or accommodation and are used once or twice daily. Their ocular tolerability is good, but they may cause respiratory and cardiac side effects [39]. PGAs increase uveoscleral aqueous outflow; they rarely cause systemic side effects but are often responsible for significant ocular adverse events (e.g. iris pigmentation changes, eyelash hypertrichosis and ocular hyperemia). PGAs have been shown to reduce IOP more than timolol, which is considered a gold standard [40-42]. In addition, they more effectively prevent IOP spikes and fluctuations over $24 \mathrm{~h}$ [43].

Second-line treatments include $\alpha$-agonists (AAs) and topical carbonic anhydrase inhibitors (CAIs), and may replace or be added to a BB or a PGA [44]. AAs decrease aqueous production and increase uveoscleral outflow, and the newer more specific $\alpha_{2}$-agonists (brimonidine and apraclonidine) cause fewer systemic hypotensive side effects than earlier nonselective AAs. Brimonidine is used for long-term therapy, whereas apraclonidine is most useful for short-term adjunctive use [43]. The major drawback of topical AAs is their frequent involvement in local allergic reactions. CAIs decrease aqueous humor production via enzymatic inhibition.

Combination drugs (e.g. $\mathrm{BB}+\mathrm{CAI}, \mathrm{BB}+\mathrm{AA}$ or $\mathrm{BB}+$ PGA) may be used to simplify dosage, improve compliance, prevent washout effect and reduce exposure to topical preservatives.

Cholinergic agonists lower IOP by increasing conventional trabecular outflow and are third-line choices in OAG. Although effective, they cause significant side effects such as induced myopia, headache, cataract formation and miosis, which can impair $\mathrm{ONH}$ and visual field evaluation, and they need 3 or 4 instillations per day. Mi-

Glaucoma: Present Challenges and Future

Trends otics (in this particular case also $\alpha$-blockers such as dapiprazole) are specifically indicated to prevent angle closure in case of narrow angle before and sometimes also after Nd:YAG iridotomy and laser iridoplasty.

Oral CAIs may be added to the regimen if the IOP is uncontrolled with topical agents or if acute IOP elevations occur, as in acute angle-closure glaucoma. They are rapidly reversible and can usefully replace topical drugs in case of intolerance, particularly in view of possible surgery. On the other hand, oral CAIs have untoward side effects such as lethargy, loss of appetite, nephrolithiasis and peripheral paresthesias.

Despite a number of pharmacological options, failure of medical treatment is a significant issue owing to drug ineffectiveness and intolerance, but also to poor patient compliance and persistence. Other causes of failure of medical therapy include lack of diurnal control, delayed efficacy and systemic side effects $[45,46]$.

On average, patients treated in a managed care setting seem to receive their eyedrop glaucoma therapy only on 7 out of 10 days [47]. Questionnaire studies reveal that up to $44 \%$ of the patients declare to miss 2 or more drop administrations per week [48], but patient statements about their adherence are not reliable and electronic medication monitor data are more deceiving: with pilocarpine (undoubtedly rather uncomfortable), only $76 \%$ of the prescribed doses were instilled but patients declared to have instilled $97 \%$ of them [49]. Topical drug class and dosage regimens do not substantially modify this reality: a personal experience was that $12 \%(0-60 \%)$ of the administrations of a single evening PGA drop were missed in a month and $22 \%$ of the patients missed over $30 \%$ of the doses, although over $90 \%$ of the subjects stated that they never missed a dose [50]. Nonadherence simulates treatment ineffectiveness and is surely a relevant factor of progression. Moreover, many patients, particularly elderly ones, are unable to correctly instill drops. This makes patient education crucial for the success of medical therapy, but also stimulates research of alternative ophthalmic drug delivery systems, such as drug-releasing contact lenses, injectable biodegradable micro- and nanoparticles, and surgically implanted devices (erodible drug implants and mini-drug pumps) [51]. Drug-eluting punctal plugs for sustained PGA release are in late stages of clinical testing and an intracameral preservative-free PGA implant is under investigation in a phase I clinical trial.

Adherence to controls and persistence in therapy is also a significant concern for the success and cost-effectiveness of medical therapy [52]. Over $50 \%$ of patients in a health maintenance organization did not refill their ini- 
tial eyedrop prescription by 1 year after diagnosis [53], and among participants in a US government health plan, $25 \%$ of newly-diagnosed OAG subjects never filled a second prescription despite the minimal cost of obtaining the drug [54].

New molecules are being evaluated in clinical trials, but robust results are not yet available: rho-associated protein kinase (ROCK) inhibitors are a promising class of novel IOP-lowering drugs. ROCK inhibitors interact with the cytoskeleton, reducing conventional aqueous outflow resistance. Topical administration of ROCK inhibitors is currently limited by significant local side effects such as hyperemia and subconjunctival hemorrhage. The development of prodrugs able to penetrate through the cornea into the anterior chamber where they convert into active compounds may overcome these side effects.

Adenosine receptor agonists are also able to increase conventional aqueous outflow and are being investigated as IOP-lowering agents. Four adenosine receptors have been cloned: A1, A2A, A2B and A3. Adenosine agonists may induce cell shrinkage and secretion of metalloproteases in human trabecular meshwork, resulting in remodeling of the extracellular matrix and reduced outflow resistance.

Cannabinoids are also being evaluated. The evidence for their use is based on the observation that smoking marijuana transiently lowers IOP. In preclinical studies, topical selective cannabinoid receptor 1 agonists lowered IOP by reducing aqueous production. Oral palmitoylethanolamide, an endogenous fatty acid ethanolamide acting on cannabinoid-like receptors, was shown to reduce IOP as an adjunctive treatment to topical hypotensive drugs.

Latrunculins are macrolides from marine sponges that inhibit actin polymerization. Studies in cynomolgus monkeys and enucleated and cadaver human eyes demonstrated that latrunculin B increases trabecular outflow through actin cytoskeleton disruption, resulting in increased openings between inner Schlemm's canal wall cells and between the canal and trabecular collagen beams. Their performance in clinical trials is limited by poor solubility and transient side effects such as increase in corneal thickness and corneal endothelial permeability, as well as induction of anterior chamber flare $[55,56]$.

\section{Nonhypotensive Therapies}

Prevention of neuronal death or deterioration was termed neuroprotection [57]. Different compounds, including antioxidants, $\mathrm{N}$-methyl-D-aspartate receptor antagonists, inhibitors of glutamate release, calcium channel blockers, polyamine antagonists and nitric synthetase inhibitors, as well as cannabinoids, aspirin, melatonin and vitamin B12, have been reported to have a neuroprotective activity [58-60].

Neuroprotection for glaucoma refers to any intervention meant to protect the optic nerve or prevent the death of retinal ganglion cells. It can affect cellular factors derived from the optic nerve itself or eliminate risk factors external to the nerve. Although significant evidence from preclinical studies has suggested a potential role of neuroprotectors in preventing glaucomatous degeneration, clinical research on the neuroprotective effects of oral and topical medical therapy for OAG in adults failed to demonstrate it. Moreover, the nonsignificant findings of a recent expensive and time-consuming phase III trial of memantine are likely to discourage other long-term trials on neuroprotection in the near future [61].

\section{Glaucoma Surgery}

Considering the issues of quality of life and poor adherence to topical therapy, as well as the very low risk profile of parasurgical treatments, we expect a trend towards an earlier use of lasers in OAG: growing evidence is being gathered to justify selective laser trabeculoplasty as an initial treatment for OAG, since it seems to approach the hypotensive power of a topical PGA [62]. Other laser sources under investigation for this purpose include micropulse diode laser trabeculoplasty, which seems somehow less efficient than argon laser trabeculoplasty (ALT) [63], and titanium sapphire laser trabeculoplasty, which seems at least as effective as ALT [64].

Despite lasers and a number of present and future ocular hypotensive agents that are likely to reduce the overall demand for antiglaucoma surgery, some cases will always require an incisional solution, both because some patients cannot achieve target pressures with maximum tolerated medical therapy and because more and more patients become intolerant to eyedrops due to a heavier exposure to topical drugs and preservatives, in terms of number of preparations and duration of medical treatment.

Since the mid-1990s, the global number of glaucoma operations has decreased thanks to the introduction of new drugs, particularly in western countries [65], but such a trend seems to have stopped and maybe reversed more recently [66]. In parallel, a more prolonged and intensive exposure to topical medications and preservatives induces a worse condition of the ocular surface of the eyes candidates for surgery [67], making intra- and/or postoperative antimetabolite use more and more common [68]. 
These facts, along with a growing patient demand for perfection and unreal expectations of outstanding visual outcomes even in desperate cases, lead to a higher specialization of glaucoma surgeons with a concentration of most procedures in larger referral centers.

All surgical treatments for glaucoma are aimed at reducing IOP, and this can be achieved through two alternative strategies: the reduction of aqueous inflow (cyclodestruction) or the enhancement of aqueous outflow (external or internal filtration surgery). According to practice guidelines $[69,70]$, the latter strategy is the first choice when medical and laser therapy are unable to achieve target IOP in seeing eyes, both because it is somehow more physiological and because the results are more definitive, titratable and stable over time.

The most popular technique for cyclodestruction is diode laser transscleral cyclophotocoagulation (CPC). Its effectiveness is hard to quantify due to heterogeneous inclusion criteria, definitions of success, follow-up times and number of retreatments, but success rates from 37 to $80 \%$ have been reported after follow-ups ranging from 10 to 28 months. Endoscopic diode laser CPC, probably due to a higher precision of treatment, has higher qualified success rates, up to $95 \%$ at 2 years [71]. Unfortunately, long-term assessment of visual acuity shows that up to $30 \%$ of treated eyes can lose 2 or more lines of vision [72] and several studies show poorer visual outcomes of CPC compared to drainage devices. Therefore, CPC is used in patients with failed drainage device implantation and/or in eyes without useful vision. Nevertheless, there may be a bias in that most studies of CPC are conducted on eyes with advanced disease and secondary refractory glaucomas. A retrospective analysis shows that the impact of CPC may not be worse than surgery in eyes with good visual acuity [73]. This suggests a possible expansion of the indications for $\mathrm{CPC}$ as an earlier treatment, for instance in elderly people with contraindications to surgery, or in countries with low hygienic standards and difficulties in managing demanding follow-ups. A new micropulse diode laser source has also recently been proposed for transscleral CPC, with an $80 \%$ success in 40 eyes with refractory glaucoma, with no further visual reduction at 18 months [74].

Aqueous outflow may be enhanced by establishing an external filtration, which is the purpose of conventional techniques, or by promoting internal filtration, and this option is being increasingly explored, particularly with microinvasive procedures that represent interesting proposals for the future.

Among conventional external filtering operations, despite several evolutions and variations proposed since its introduction in the 1960s, trabeculectomy (TRAB) is still the most commonly performed antiglaucomatous surgical procedure and must be considered a gold standard [75]. In the 1990s, TRAB has been augmented by the introduction of antimetabolites, in particular mitomycin C (MMC) and 5-fluorouracil; MMC is more potent and is indicated in eyes at higher failure risk, and 5-fluorouracil is commonly used also postoperatively to reduce scarring when blebs show a tendency towards failure.

Unfortunately, surgical outcomes are generally assessed in terms of IOP control, which must be accepted as a surrogate indicator of success. A recent literature review reveals that among 100 studies of TRAB, 99 report no information about optic nerve morphology or function across the follow-up. Moreover, even concerning the only statement of IOP control, the same review found 92 distinct IOP-related definitions of success, with reported 3 -year success rates ranging from 36 to $98 \%$. This, along with the differences in inclusion criteria, makes comparisons among studies practically impossible and also raises some medicolegal concerns about the figures that should be indicated in consent forms [76].

Many stylistic variations regarding conjunctival wound placement and flap size and shape do not substantially affect the final outcome of TRAB [77]. With MMC, early bleb leaks are more critical with fornix-based flaps, whereas late bleb leaks and infection seem more common with limbus-based flaps [78]. In fact, the conjunctival flap used to be fornix-based before the introduction of antimetabolites, then it was more commonly limbus-based to reduce the risk of leaks, and nowadays it tends again to be fornixbased to enhance posterior aqueous diffusion. MMC use, which is very common, increases the tonometric success of TRAB but also the rate of postoperative complications, as if amplifying the surgical imperfections, and therefore requires a very accurate technique. New wound-healing modulators under clinical investigation for trabeculectomy are anti-VEGF agents and collagen matrix implants (OloGen), but the latter did not show significant advantages in a pilot study [79].

The ultimate evolution of TRAB, the so-called safe TRAB technique, performed using a fornix-based conjunctival flap, an anterior chamber maintainer, a standardized punch technique, and a combination of adjustable and releasable sutures, achieved an IOP $<21 \mathrm{~mm} \mathrm{Hg}$ in $100 \%$ of the cases in a low-to-moderate failure risk series of 56 eyes (mostly without antimetabolite use), but there still was 1 case of flat anterior chamber requiring surgery, 1 hypotony lasting over 3 weeks, 1 hyphema, 1 malignant glaucoma, 5 cases of choroidals, 3 cases of 
corneal epitheliopathy or erosion and 1 endophthalmitis [80]. Even in the hands of the inventors themselves, the same technique with MMC and aggressive postoperative management performed on high-risk subjects achieved a 3 -year survival rate for IOP $\leq 21 \mathrm{~mm} \mathrm{Hg}$ with or without medications of $86 \%$, with $15 \%$ choroidals and an $8.5 \%$ occurrence of early bleb leaks requiring resuturing [81].

Several other techniques involving external filtration have been proposed and introduced in the endeavor to enhance success and achieve a long-lasting IOP control with unmedicated low-teen IOPs, but also especially to reduce the risk of sight-threatening complications of TRAB such as hypotony, choroidals, hemorrhage and particularly endophthalmitis. The last cumulatively affects $0.2-1.5 \%$ of eyes undergoing simple superior TRAB, and $0.8-4 \%$ of cases operated with antimetabolites (with an incidence up to $2.5 \%$ per patient-year), with a much higher risk (up to over 13\%) in eyes with inferior blebs [82] and a life-long persistency of risk [83].

An interesting augmentation of TRAB is represented by the implantation of the Ex-PRESS mini-drainage device under a trabeculectomy-style scleral flap: this does not change the nature of the filtering operation but avoids the need for iridectomy and allows to displace the site of aqueous drainage a little inside the anterior chamber. ExPRESS produces a calibrated fistulization, but this is not a key advantage in the opinion of the authors because the main resistance to aqueous outflow is in any case provided by the scleral flap, quite independently of the caliber of the internal sclerectomy. In a long-term randomized comparison with standard TRAB (without MMC), the Ex-PRESS implant significantly increased the absolute success of the filter for the first 3 postoperative years and reduced the number of medications needed and the risk of complications for at least 5 years [84]. A small randomized trial comparing MMC Ex-PRESS implantation versus MMC, TRAB revealed a similar tonometric success with lower complication rates for the former [85]. In our opinion, ExPRESS implantation, which is a rather expensive augmentation to TRAB, is specifically indicated if an iridectomy may lead to vitreous prolapse in the anterior chamber with a risk of filter plugging (such as in traumatic or pseudoexfoliation glaucoma) and in conditions in which an obstruction may develop at the level of the internal ostium of a conventional sclerectomy but may be less likely to grow over a metal shaft and plug a drainage hole placed more internally in the anterior chamber (such as in quiescent neovascular glaucoma, Cogan-Reese syndrome, epithelial ingrowth and ICE syndromes).
Deep sclerectomy (DS) is the paradigm of the so-called nonpenetrating glaucoma surgery (NPGS) and is a valuable option in OAG eyes naïve to previous filtering surgeries, with a 10 -year survival rate for IOP $\leq 21 \mathrm{~mm} \mathrm{Hg}$ of $45 \%$ with no medication and $78 \%$ with or without medication. Nd:YAG laser goniopuncture is required in over half of the cases to control IOP in the course of the followup, virtually making the procedure penetrating at last [86]. A recent meta-analysis reveals that, in terms of global success rates, DS was inferior to TRAB, but the percentage of cases achieving target IOP without medication at 4 years was $64.6 \%$ for DS with implant and 52.1\% for MMC DS compared to $70 \%$ for MMC TRAB. These data suggest that if DS is associated with the use of antimetabolites and/or implants and/or goniopuncture, it can provide success rates that are substantially equivalent to those of MMC TRAB, with a lower incidence of complications such as shallow anterior chamber (11 vs. $22.4 \%$ ), hypotony ( 8.2 vs. $20.4 \%)$ and choroidal detachment (11.6 vs. $19.3 \%)$ for MMC DS and MMC TRAB, respectively [87]. This justifies our choice to favor NPGS with implant and/ or MMC as an alternative to TRAB in selected cases of OAGs naïve to previous surgery.

Lasers have been proposed to realize precise and standardized tissue ablations in penetrating and nonpenetrating filtering surgeries. The clinical success of the former was deceiving, mainly because of thermal damage and healing response that quickly obliterated laser-created transscleral fistulae, but possible applications for NPGS are more promising [88]. In fact, a $\mathrm{CO}_{2}$ laser has been used to ablate in a very reproducible manner the inner flap of DS in a procedure that was named CLASS $\left(\mathrm{CO}_{2}\right.$ laser-assisted sclerectomy); the ablation stops as soon as aqueous starts percolating through the trabeculodescemetic window, avoiding ruptures and leaving a very even, thin and smooth deep scleral layer. Presently, only 1 pilot study of CLASS has been published, on 37 patients, most of whom were also exposed to MMC intraoperatively. Complete success at 6 and 12 months was not outstanding, attaining only 76.7 and $60 \%$, respectively, but no major complications ensued, and this aspect can be valuable in skipping the learning curve that otherwise affects incisional NPGSs [89].

Glaucoma drainage devices are gaining increasing favor in recent years even in nonrefractory glaucomas and are likely to become more popular in the future. A recent report of a large prospective study comparing Baerveldt implantation with MMC TRAB in eyes with previous cataract extraction and/or failed filtering surgery revealed a 5-year cumulative probability of failure (despite medication) of 29.8 versus $46.9 \%$ and a rate of reoperation of 9 versus $29 \%$ 
for the two procedures, respectively [90]. Tube patients used more hypotensive drugs for the first 2 years, but the difference in medication equalized in the following years. MMC TRAB eyes had more frequent early complications, whereas tube eyes displayed a nonsignificantly higher risk of late complications and/or vision loss and of consequent reoperation to resolve them [91]. In our opinion, TRAB or DS are still the first-line surgical options in cases at low risk of failure (being quicker, less invasive and cheaper) whereas glaucoma drainage devices are indicated in high-risk eyes or in failed multiple or revised MMC TRAB (in which tube exposure or endothelial decompensation requiring further surgery over time are acceptable risks).

Conventional drainage procedures with external filtration will surely represent the bulk of glaucoma surgeries for years, but some internal filtration procedures aimed at restoring physiological aqueous outflow independent of possible accessorial external filtration, which at present play a marginal role in the surgical armamentarium, are promising and must be considered with interest for some specific applications.

Internal filtration procedures are substantially indicated only in OAG or pseudophakic eyes and require a functional integrity of the distal outflow system which can be assessed by provocative gonioscopy (eliciting blood reflux) and by fluorescein channelography. In these techniques, the absence or independence of bleb formation avoids most of the complications that characterize external filtering operations, making them safer, but their value must also be measured in terms of IOP reduction. The absence of external filtration also reduces visual disturbances, astigmatism and patient discomfort and requires less follow-up visits and chair time postoperatively. Moreover, the resistance of the distal physiological outflow pathway and the episcleral venous pressure minimize the risk of hypotony. These surgical options are likely to become more diffuse in the near future, specifically in early OAGs with high IOPs, younger and active patients, contact lens wearers and subjects intolerant to eyedrops, and when the target IOP is not very low.

$\mathrm{Ab}$ externo procedures include viscocanalostomy (VC) and canaloplasty $(\mathrm{CP})$, both of which require a learning curve that lowers initial success rates. Although VC in association with phacoemulsification was reported to be as effective as MMC TRAB at 1 year [92], it is nowadays less employed since randomized trials indicate that $\mathrm{VC}$ alone is less effective even than simple TRAB in terms of IOP reduction [93] and that its efficacy tends to fade more rapidly over time [94]. CP shares the same mechanism of action as VC, but is more effective thanks to its involvement

Glaucoma: Present Challenges and Future

Trends of the whole circumference of Schlemm's canal and to sustained deformation induced by a tension suture. In a comparison between the two techniques, success (unmedicated IOP $\leq 17 \mathrm{~mm} \mathrm{Hg}$ at 3 years) was obtained in $83 \%$ with $\mathrm{CP}$ versus $42 \%$ in VC [95]. A multicenter study assessing safety and efficacy of CP revealed a sustained IOP reduction from a baseline of $23.2 \mathrm{~mm} \mathrm{Hg}$ on a mean of $1.8 \mathrm{med}-$ ications to $15.2 \mathrm{~mm} \mathrm{Hg}$ on 0.8 medications at 3 years [96], and a long-term study on 60 eyes of African patients (at higher risk of failure) showed a $77.5 \%$ survival of IOP $<21$ $\mathrm{mm} \mathrm{Hg}$ without medication at 36 months [97].

Finally, the so-called microinvasive glaucoma surgeries consist of procedures that enhance physiological aqueous outflow but are performed ab interno with minimal incisions. These are surely less effective than conventional filtering procedures but are also characterized by minimal manipulation and complications.

The iStent (Glaukos Corporation, Laguna Hills, Calif., USA) is an ' $L$ '-shaped titanium stent that is introduced in Schlemm's canal (best in pseudophakic eyes and possibly in concomitance with cataract surgery) through the angle region, thus allowing bypassing of an increased trabecular resistance. Several studies confirm the safety and the effectiveness, although not dramatic, of the device [98]. A multicenter clinical trial randomized $240 \mathrm{OAG}$ eyes to either phacoemulsification + iStent or phacoemulsification alone, achieving a 1 -year unmedicated IOP $\leq 21$ in 72 versus $50 \%$ of the cases, respectively, with no substantial differences in adverse events [99]. New designs are being tested for further iStent generations, and multiple devices can be implanted, although this opportunity seems neither very practical nor cost-effective.

The Hydrus device (Ivantis Inc., Irvine, Calif., USA) is an 8-mm-long nitinol Schlemm's canal scaffold with 3 openings that is inserted in the canal, producing a significant increase in outflow facility compared to controls [100]. A 6-month pilot clinical study involving 37 subjects with primary OAG showed promising results: mean IOP dropped from $21.6 \mathrm{~mm} \mathrm{Hg}$ on 1.7 glaucoma medications to $16.9 \mathrm{~mm} \mathrm{Hg}$ on 0.6 medications on average [101].

The CyPass (Transcend Medical, Menlo Park, Calif., USA) is a polyamide tube that is inserted in the suprachoroidal space through the angle region. It has been reported to reduce IOP by $37 \%$ in medically uncontrolled eyes, with more than a $50 \%$ reduction in glaucoma medications at 6 months when implanted in 57 eyes in concomitance with phacoemulsification [102].

Trabectome is a high-frequency electrocautery that removes and aspirates a strip of trabecular meshwork and inner wall of Schlemm's canal, and can be used alone or 
in concomitance with cataract surgery. Many studies have investigated the effectiveness and safety of the procedure [98]. In 2010, a report of 538 Trabectome and 290 phaco-Trabectome procedures revealed a 1-year qualified success (IOP $<21 \mathrm{~mm} \mathrm{Hg}$ ) of $64.9 \%$ for the former and $86.9 \%$ for the latter, with a significant drop in the number of medications [103]. A retrospective cohort study comparing Trabectome with MMC TRAB demonstrated a 2 -year qualified success (IOP $\leq 21 \mathrm{~mm} \mathrm{Hg}$ ) of 22.4 versus $76.1 \%$, respectively, with a relevant difference in the final number of medications in favor of MMC TRAB. Hyphema, spontaneously reabsorbed within 7 days, occurred in $100 \%$ of the patients in the study group and in $2.9 \%$ of the MMC TRAB group. Early IOP spikes occurred in approximately $3 \%$ of the cases in both groups, but other complications were seen only in the MMC TRAB group [104]. Another study demonstrated that a previously failed Trabectome procedure does not negatively impact the outcome of subsequent TRAB, and this is crucial to support the choice of submitting selected patients to a surgical option that is notably less effective for the sake of safety, without affecting the success rates of future conventional filtration surgery if needed [105].

Finally, excimer laser trabeculostomy creates small perforations through the trabecular meshwork and the inner wall of Schlemm's canal using the energy delivered ab interno through a fiberoptic probe and proved substantially equivalent to selective laser trabeculoplasty in a 2-year prospective randomized comparative trial [106].

\section{Conclusions}

Despite the number of glaucomatous patients, no case is equal to another, particularly with regard to the unique coupling between a disease with multiform origins and manifestations and single-patient personality, condition, age, needs and social state. Communication of diagnosis, patient education, management of possible visual disability, and therapeutic approach are necessarily customized. This is a difficulty but also an enrichment for our everyday practice, which is never monotonous. Clinical facts are science, but the interaction between patient and physician is somewhat of an art, and success in curing disease comes from competent application of technique, rationality and guidelines, but is also a gift. The state of the art of glaucoma management is in constant evolution: starting from it, we wished to discuss some major challenges we are aware of and the possible developments they will bring about. This review is surely partial and is meant just to stimulate further debate, particularly among those who are never fully satisfied and are eager to search out new solutions. We have no doubt that in the next 10 years our practice will change and improve: some evolutions can be anticipated and some cannot even yet be thought of. For sure our work will be more and more interesting and successful: we have great hope in the future!

\section{References}

1 Resnikoff S, Pascolini D, Etya'ale D, et al: Global data on visual impairment in the year 2002. Bull World Health Organ 2004;82:844-851.

-2 Quigley HA: Glaucoma. Lancet 2011;377: 1367-1377.

-3 Quigley HA, Broman AT: The number of people with glaucoma worldwide in 2010 and 2020. Br J Ophthalmol 2006;90:262-267.

4 Varma R, Lee PP, Goldberg I, Kotak S: An assessment of the health and economic burdens of glaucoma. Am J Ophthalmol 2011;152: 515-522.

5 Deva NC, Insull E, Gamble G, Danesh-Meyer HV: Risk factors for first presentation of glaucoma with significant visual field loss. Clin Experiment Ophthalmol 2008;36:217-221.

6 Buys YM, Gaspo R, Kwok K: Referral source, symptoms, and severity at diagnosis of ocular hypertension or open-angle glaucoma in various practices. Can J Ophthalmol 2012;47: 217-222.
Vaahtoranta-Lehtonen $\mathrm{H}$, Tuulonen $\mathrm{A}$, Aronen P, et al: Cost effectiveness and cost utility of an organized screening programme for glaucoma. Acta Ophthalmol Scand 2007; 85:508-518.

8 Nayak BK, Maskati QB, Parikh R: The unique problem of glaucoma: under-diagnosis and over-treatment. Indian J Ophthalmol 2011; 59(suppl 1):S1-S2.

-9 Mowatt G, Burr JM, Cook JA, et al: Screening tests for detecting open-angle glaucoma: systematic review and meta-analysis. Invest Ophthalmol Vis Sci 2008;49:5373-5385.

10 Burr JM, Mowatt G, Hernandez R, et al: The clinical effectiveness and cost-effectiveness of screening for open angle glaucoma: a systematic review and economic evaluation. Health Technol Assess 2007;11:1-190.

11 Siddiqui MA, Azuara-Blanco A, Burr J: The quality of reporting of diagnostic accuracy studies published in ophthalmic journals. Br J Ophthalmol 2005;89:261-265.
12 Tuulonen A: Cost-effectiveness of screening for open angle glaucoma in developed countries. Indian J Ophthalmol 2011;59(suppl): S24-S30.

13 Tuulonen A: Economics of surgery worldwide. Developed countries; in Shaarawy TM, Sherwood MB, Hitchings RA, Crowston JG (eds): Glaucoma. Philadelphia, Saunders, 2009, pp 3-11.

14 Haymes SA, LeBlanc RP, Nicolela MT, et al: Risk of falls and motor vehicle collisions in glaucoma. Invest Ophthalmol Vis Sci 2007; 48:1149-1155.

15 Haymes SA, LeBlanc RP, Nicolela MT, et al: Glaucoma and on-road driving performance. Invest Ophthalmol Vis Sci 2008;49:30353041.

16 Hatt S, Wormald R, Burr J: Screening for prevention of optic nerve damage due to chronic open angle glaucoma. Cochrane Database Syst Rev 2006;4:CD006129. 
17 Grødum K, Heijl A, Bengtsson B: A comparison of glaucoma patients identified through mass screening and in routine clinical practice. Acta Ophthalmol Scand 2002;80:627-631.

$\checkmark 18$ Reus NJ, Lemij HG, Garway-Heath DF, et al: Clinical assessment of stereoscopic optic disc photographs for glaucoma: the European Optic Disc Assessment Trial. Ophthalmology 2010;117:717-723.

19 Hood DC, Kardon RH: A framework for comparing structural and functional measures of glaucomatous damage. Prog Retin Eye Res 2007;26:688-710.

20 Weinreb RN, Zangwill LM, Jain S, et al: Predicting the onset of glaucoma: the confocal scanning laser ophthalmoscopy ancillary study to the Ocular Hypertension Treatment Study. Ophthalmology 2010;117:1674-1683.

-21 Folio LS, Wollstein G, Schuman JS: Optical coherence tomography: future trends for imaging in glaucoma. Optom Vis Sci 2012; 89:E554-E562.

22 Gong G, Kosoko-Lasaki S, Haynatzki G, et al: Inherited, familial and sporadic primary open-angle glaucoma. J Natl Med Assoc 2007; 99:559-563.

-23 Allingham RR, Liu Y, Rhee DJ: The genetics of primary open-angle glaucoma: a review. Exp Eye Res 2009;88:837-844.

24 Stone EM, Fingert JH, Alward WL, et al: Identification of a gene that causes primary open angle glaucoma. Science 1997;275:668-670.

25 Rezaie T, Child A, Hitchings R, et al: Adultonset primary open-angle glaucoma caused by mutations in optineurin. Science 2002;295: 1077-1079.

26 Monemi S, Spaeth G, DaSilva A, et al: Identification of a novel adult-onset primary openangle glaucoma (POAG) gene on 5q22.1. Hum Mol Genet 2005;14:725-733.

27 Pasutto F, Matsumoto T, Mardin CY, et al: Heterozygous NTF4 mutations impairing neurotrophin-4 signaling in patients with primary open-angle glaucoma. Am J Hum Genet 2009;85:447-456.

-28 Vithana EN, Nongpiur ME, Venkataraman $\mathrm{D}$, et al: Identification of a novel mutation in the NTF4 gene that causes primary openangle glaucoma in a Chinese population. Mol Vis 2010;16:1640-1645.

29 Fan BJ, Wang DY, Fan DS, et al: SNPs and interaction analyses of myocilin, optineurin, and apolipoprotein $\mathrm{E}$ in primary open angle glaucoma patients. Mol Vis 2005;11:625-631.

-30 Thorleifsson G, Walters GB, Hewitt AW, et al: Common variants near CAV1 and CAV2 are associated with primary open-angle glaucoma. Nat Genet 2010;42:906-909.

- 31 Burdon KP, Macgregor S, Hewitt AW, et al: Genome-wide association study identifies susceptibility loci for open angle glaucoma at TMCO1 and CDKN2B-AS1. Nat Genet 2011; 43:574-578.

32 Thorleifsson G, Magnusson KP, Sulem P, et al: Common sequence variants in the LOXL1 gene confer susceptibility to exfoliation glaucoma. Science 2007;317:1397-1400.
33 Ozaki M, Lee KY, Vithana EN, et al: Association of LOXL1 gene polymorphisms with pseudoexfoliation in the Japanese. Invest Ophthalmol Vis Sci 2008;49:3976-3980.

34 Ramprasad VL, George R, Soumittra N, et al: Association of non-synonymous single nucleotide polymorphisms in the LOXL1 gene with pseudoexfoliation syndrome in India. Mol Vis $2008 ; 14: 318-322$

35 Hayashi H, Gotoh N, Ueda Y, et al: Lysyl oxidase-like 1 polymorphisms and exfoliation syndrome in the Japanese population. Am J Ophthalmol 2008; 145:582-585.

36 Chen L, Jia L, Wang N, et al: Evaluation of LOXL1 polymorphisms in exfoliation syndrome in a Chinese population. Mol Vis 2009; 15:2349-2357.

37 Williams SE, Whigham BT, Liu Y, et al: Major LOXL1 risk allele is reversed in exfoliation glaucoma in a black South African population. Mol Vis 2010;16:705-712.

38 van Koolwijk LM, Ramdas WD, Ikram MK, et al: Common genetic determinants of intraocular pressure and primary open-angle glaucoma. PLoS Genet 2012;8:e1002611.

39 Lee DA, Higginbotham EJ: Glaucoma and its treatment: a review. Am J Health Syst Pharm 2005;62:691-699.

40 Netland PA, Landry T, Sullivan EK, et al: Travoprost compared with latanoprost and timolol in patients with open-angle glaucoma or ocular hypertension. Am J Ophthalmol 2001; 132:472-484.

41 Sherwood M, Brandt J, Bimatoprost Study Groups 1 and 2: Six-month comparison of bimatoprost once-daily and twice-daily with timolol twice-daily in patients with elevated intraocular pressure. Surv Ophthalmol 2001; 45(suppl 4):S361-S368.

42 Hedman K, Alm A, Gross RL: Pooled-data analysis of three randomized, double-masked, six-month studies comparing intraocular pressure-reducing effects of latanoprost and timolol in patients with ocular hypertension. J Glaucoma 2003;12:463-465.

43 Tsai JC, Kanner EM: Current and emerging medical therapies for glaucoma. Expert Opin Emerg Drugs 2005;10:109-118.

-44 Schwartz GF, Reardon G, Mozaffari E: Persistency with latanoprost or timolol in primary open-angle glaucoma suspects. Am J Ophthalmol 2004;137(1 suppl):S13-S16.

45 Asrani S, Zeimer R, Wilensky J, et al: Large diurnal fluctuations in intraocular pressure are an independent risk factor in patients with glaucoma. J Glaucoma 2000;9:134-142.

46 Bergea B, Bodin L, Svedbergh B: Impact of intraocular pressure regulation on visual fields in open-angle glaucoma. Ophthalmology 1999;106:997-1004.

47 Gurwitz JH, Yeomans SM, Glynn RJ, et al: Patient noncompliance in the managed care setting. The case of medical therapy for glaucoma. Med Care 1998:36:357-369.
48 Konstas AG, Maskaleris G, Gratsonidis S, Sardelli C: Compliance and viewpoint of glaucoma patients in Greece. Eye (Lond) 2000;14: 752-756.

-49 Kass MA, Meltzer DW, Gordon M, et al: Compliance with topical pilocarpine treatment. Am J Ophthalmol 1986;101:515-523.

50 Carassa RG, Bettin P, Fiori M, Ciampi C: Are glaucoma patients reliable in managing their therapy? Invest Ophthalmol Vis Sci 2007;48: 5582, E-abstract.

51 Lavik E, Kuehn MH, Kwon YH: Novel drug delivery systems for glaucoma. Eye (Lond) 2011;25:578-586.

52 Kosoko O, Quigley HA,Vitale S, et al: Risk factors for noncompliance with glaucoma follow-up visits in a residents' eye clinic. Ophthalmology 1998;105:2105-2111.

-53 Fiscella RG, Green A, Patuszynski DH, Wilensky J: Medical therapy cost considerations for glaucoma. Am J Ophthalmol 2003; 136:18-25

54 Gurwitz JH, Glynn RJ, Monane M, et al: Treatment for glaucoma: adherence by the elderly. Am J Public Health 1993;83:711-716.

55 Chen J, Runyan SA, Robinson MR: Novel ocular antihypertensive compounds in clinical trials. Clin Ophthalmol 2011;5:667-677.

56 Zhang K, Zhang L, Weinreb RN: Ophthalmic drug discovery: novel targets and mechanisms for retinal diseases and glaucoma. Nat Rev Drug Discov 2012;11:541-559.

57 Levin LA: Direct and indirect approaches to neuroprotective therapy of glaucomatous optic neuropathy. Surv Ophthalmol 1999; 43(suppl 1):S98-S101.

58 Neacsu A, Oprean C, Curea M, et al: Neuroprotection with carotenoids in glaucoma (in Romanian). Oftalmologia 2003;59:70-75.

59 Neufeld AH: New conceptual approaches for pharmacological neuroprotection in glaucomatous neuronal degeneration. J Glaucoma 1998;7:434-438.

60 Weinreb RN, Levin LA: Is neuroprotection a viable therapy for glaucoma? Arch Ophthalmol 1998;117:1540-1544.

61 Sena DF, Ramchand K, Lindsley K: Neuroprotection for treatment of glaucoma in adults. Cochrane Database Syst Rev 2010; 2:CD006539.

62 Katz LJ, Steinmann WC, Kabir A, et al: Selective laser trabeculoplasty versus medical therapy as initial treatment of glaucoma: a prospective, randomized trial. J Glaucoma 2012 21:460-468.

63 Detry-Morel M, Muschart F, Pourjavan S: Micropulse diode laser $(810 \mathrm{~nm})$ versus argon laser trabeculoplasty in the treatment of open-angle glaucoma: comparative shortterm safety and efficacy profile. Bull Soc Belge Ophtalmol 2008;308:21-28

64 Goldenfeld M, Melamed S, Simon G, Ben Simon GJ: Titanium:sapphire laser trabeculoplasty versus argon laser trabeculoplasty in patients with open-angle glaucoma. Ophthalmic Surg Lasers Imaging 2009;40:264-269. 
65 Rachmiel R, Trope GE, Chipman ML, et al: Effect of medical therapy on glaucoma filtration surgery rates in Ontario. Arch Ophthalmol 2006;124:1472-1477.

66 Keenan TD, Wotton CJ, Goldacre MJ: Recent trends in the rate of trabeculectomy in England. Eye (Lond) 2011;25:1243-1245.

-67 Baudouin C: Ocular surface and external filtration surgery: mutual relationships. De Ophthalmol 2012;50:64-78.

68 Holló G: Wound healing and glaucoma surgery: modulating the scarring process with conventional antimetabolites and new molecules. Dev Ophthalmol 2012;50:79-89.

69 European Glaucoma Society: Terminology and Guidelines for Glaucoma, ed 3. 2009.

70 American Academy of Ophthalmology: Primary Open-Angle Glaucoma, Preferred Practice Pattern. San Francisco, American Academy of Ophthalmology, 2005, pp 15-16.

71 Lin SC: Endoscopic and transscleral cyclophotocoagulation for the treatment of refractory glaucoma. J Glaucoma 2008;17:238-247.

72 Kosoko O, Gaasterland DE, Pollack IP, Enger CL: Long-term outcome of initial ciliary ablation with contact diode laser transscleral cyclophotocoagulation for severe glaucoma. The Diode Laser Ciliary Ablation Study Group. Ophthalmology 1996;103:1294-1302.

-73 Rotchford AP, Jayasawal R, Madhusudhan S, et al: Transscleral diode laser cycloablation in patients with good vision. Br J Ophthalmol 2012;94:1180-1183.

-74 Tan AM, Chockalingam M, Aquino MC, et al: Micropulse transscleral diode laser cyclophotocoagulation in the treatment of refractory glaucoma. Clin Experiment Ophthalmol 2010;38:266-272.

75 Khaw PT, Chiang M, Shah P, et al: Enhanced trabeculectomy: the Moorfields safer surgery system. Dev Ophthalmol 2012;50:1-28.

-76 Rotchford AP, King AJ: Moving the goal posts definitions of success after glaucoma surgery and their effect on reported outcome. Ophthalmology 2010;117:18-23.

-77 Shuster JN, Krupin T, Kolker AE, Becker B: Limbus- $v$ fornix-based conjunctival flap in trabeculectomy. A long-term randomized study. Arch Ophthalmol 1984;102:361-362.

$\checkmark 78$ Wells AP, Cordeiro MF, Bunce C, Khaw PT: Cystic bleb formation and related complications in limbus- versus fornix-based conjunctival flaps in pediatric and young adult trabeculectomy with mitomycin C. Ophthalmology 2003;110:2192-2197.

79 Papaconstantinou D, Georgalas I, Karmiris E, et al: Trabeculectomy with OloGen versus trabeculectomy for the treatment of glaucoma: a pilot study. Acta Ophthalmol 2010;88:80-85.

80 Stalmans I, Gillis A, Lafaut AS, Zeyen T: Safe trabeculectomy technique: long-term outcome. Br J Ophthalmol 2006;90:44-47.
81 Shah P, Agrawal P, Khaw PT, et al: ReGAE 7: long-term outcomes of augmented trabeculectomy with mitomycin $\mathrm{C}$ in African-Caribbean patients. Clin Experiment Ophthalmol 2012;40:e176-e182.

82 Prasad N, Latina MA: Blebitis and endophthalmitis after glaucoma filtering surgery. Int Ophthalmol Clin 2007;47:85-97.

83 Ciulla TA, Beck AD, Topping TM, Baker AS: Blebitis, early endophthalmitis, and late endophthalmitis after glaucoma filtering surgery. Ophthalmology 1997;104:986-995.

84 De Jong L, Lafuma A, Aguadé AS, Berdeaux G: Five-year extension of a clinical trial comparing the Ex-PRESS glaucoma filtration device and trabeculectomy in primary open-angle glaucoma. Clin Ophthalmol 2011;5:527533.

85 Sugiyama T, Shibata M, Kojima S, et al: The first report on intermediate-term outcome of Ex-PRESS glaucoma filtration device implanted under scleral flap in Japanese patients. Clin Ophthalmol 2011;5:1063-1066.

86 Bissig A, Rivier D, Zaninetti M, et al: Ten years follow-up after deep sclerectomy with collagen implant. J Glaucoma 2008; 17:680686.

87 Cheng JW, Cheng SW, Cai JP, et al: Systematic overview of the efficacy of nonpenetrating glaucoma surgery in the treatment of open angle glaucoma. Med Sci Monit 2011;17:155163.

88 Geffen N, Assia EI, Melamed S: Laser-assisted techniques for penetrating and nonpenetrating glaucoma surgery. Dev Ophthalmol 2012; 50:96-108.

89 Geffen N, Ton Y, Degani J, Assia EI: $\mathrm{CO}_{2}$ laser-assisted sclerectomy surgery, part II: multicenter clinical preliminary study. J Glaucoma 2012;21:193-198.

90 Gedde SJ, Schiffman JC, Feuer WJ, et al: Treatment outcomes in the Tube Versus Trabeculectomy (TVT) study after five years of follow-up. Am J Ophthalmol 2012;153:789803.

91 Gedde SJ, Herndon LW, Brandt JD, et al: Postoperative complications in the Tube Versus Trabeculectomy (TVT) study during five years of follow-up. Am J Ophthalmol 2012; 153:804-814

92 Kobayashi H, Kobayashi K: Randomized comparison of the intraocular pressure-lowering effect of phacoviscocanalostomy and phacotrabeculectomy. Ophthalmology 2007; 114:909-914.

93 Carassa RG, Bettin P, Fiori M, Brancato R: Viscocanalostomy versus trabeculectomy in white adults affected by open-angle glaucoma: a 2-year randomized, controlled trial. Ophthalmology 2003;110:882-887.
94 Gilmour DF, Manners TD, Devonport H, et al: Viscocanalostomy versus trabeculectomy for primary open angle glaucoma: 4-year prospective randomized clinical trial. Eye (Lond) 2009;23:1802-1807.

-95 Peckar CO, Koerber N: Canaloplasty for open angle glaucoma: a three-year critical evaluation and comparison with viscocanalostomy. Spektrum Augenheilkd 2008;22: 240-246.

\$6 Lewis RA, von Wolff $\mathrm{K}$, Tetz M, et al: Canaloplasty: three-year results of circumferential viscodilation and tensioning of Schlemm canal using a micro catheter to treat open-angle glaucoma. J Cataract Refract Surg 2011;37:682-690.

-97 Grieshaber MC, Pienaar A, Olivier J, Stegmann R: Canaloplasty for primary open-angle glaucoma: long-term outcome. $\mathrm{Br} \mathrm{J} \mathrm{Oph-}$ thalmol 2010;94:1478-1482.

98 Francis BA, Winarko J: Ab interno Schlemm's canal surgery: Trabectome and iStent. Dev Ophthalmol 2012;50:125-136.

-99 Samuelson TW, Katz LJ, Wells JM, et al: Randomized evaluation of the trabecular micro-bypass stent with phacoemulsification in patients with glaucoma and cataract. Ophthalmology 2011;118:459-467.

100 Camras LJ, Yuan F, Fan S, et al: A novel Schlemm's Canal scaffold increases outflow facility in a human anterior segment perfusion model. Invest Ophthalmol Vis Sci 2012; 53:6115-6121.

101 Samuelson TW, Pfeiffer N, Lorenz K: Sixmonth results from a prospective, multicenter study of a nickel-titanium Schlemm's canal scaffold for IOP reduction in open-angle glaucoma. 22nd Annual AGS Meeting, New York, March 2012.

102 Hoeh H, Ahmed II, Grisanti S, et al: Early postoperative safety and surgical outcomes after implantation of a suprachoroidal micro-stent for the treatment of open-angle glaucoma concomitant with cataract surgery. J Cataract Refract Surg 2013;39:431437.

103 Mosaed S, Rhee DJ, Filippopoulos T, et al: Trabectome outcomes in adult open-angle glaucoma patients: one year follow-up. Clin Surg Ophthalmol 2010;28:5-9.

104 Jea SY, Francis BA, Vakili G, et al: Ab interno trabeculectomy versus trabeculectomy for open-angle glaucoma. Ophthalmology 2012;119:36-42.

105 Jea SY, Mosaed S, Vold SD, Rhee DJ: Effect of a failed Trabectome on subsequent trabeculectomy. J Glaucoma 2012;21:71-75.

106 Babighian S, Caretti L, Tavolato M, et al: Excimer laser trabeculotomy vs 180 degrees selective laser trabeculoplasty in primary open-angle glaucoma. A 2-year randomized, controlled trial. Eye (Lond) 2010;24:632638 . 